UDK 519.21

\author{
O. A. Yarova, Ya. I. Yeleyko
}

\title{
THE RENEWAL EQUATION IN NONLINEAR APPROXIMATION
}

O. A. Yarova, Ya. I. Yeleyko. The renewal equation in nonlinear approximation, Mat. Stud. 56 (2021), 103-106.

The family of Markov processes are considered. We study the multidimensional renewal equation in nonlinear approximation. The purpose of the paper is to find the limit of renewal function.

Let $X^{\varepsilon}(t)$ be a family of Markov processes with continuous time and a finite number of states $1,2, . ., n$, and $X^{\varepsilon}(t) \rightarrow X(t)$, where $\varepsilon \rightarrow 0$.

Suppose that $\xi_{i}^{\varepsilon}(t)$ is a process with independent increments, $t \geq 0, \xi_{i}^{\varepsilon}(t)>0, i=$ $1,2, . ., n$. We consider the following process

$$
\zeta^{\varepsilon}(t)= \begin{cases}\xi_{i(1)}^{\varepsilon}(t), & \text { if } t<\tau, X^{\varepsilon}(0)=i \\ \xi_{i(1)}^{\varepsilon}(\tau)+\xi_{j(2)}^{\varepsilon}(t-\tau), & \text { if } \tau \leq t<\tau_{1}, X^{\varepsilon}(\tau)=j \\ \xi_{i(1)}^{\varepsilon}(\tau)+\xi_{j(2)}^{\varepsilon}\left(\tau_{1}-\tau\right)+\xi_{s(3)}^{\varepsilon}\left(t-\tau_{1}\right), & \text { if } \tau_{1} \leq t<\tau_{2}, X^{\varepsilon}\left(\tau_{2}\right)=s \\ \vdots & \end{cases}
$$

In this representation $\xi_{i(p)}^{\varepsilon}(t)$ are independent copies of $\xi_{i}^{\varepsilon}(t)$.

Consider the next conditional expectation $E\left(e^{-\zeta^{\varepsilon}(t)} \mid X^{\varepsilon}(0)=i\right)=E_{i}\left(e^{-\zeta^{\varepsilon}(t)}\right)$.

This implies

$$
E_{i}\left(e^{-\zeta^{\varepsilon}(t)}\right)=E_{i}\left(e^{-\zeta^{\varepsilon}(t)}, \tau>t\right)+\sum_{j=1}^{n} \int_{0}^{t} E_{i}\left(e^{-\lambda \zeta^{\varepsilon}(t)}, \tau \in d u, X^{\varepsilon}(\tau)=j\right) \cdot E_{j}\left(e^{-\lambda \zeta^{\varepsilon}(t-u)}\right) .
$$

Then

$$
E_{i}\left(e^{-\lambda \xi^{\varepsilon}(u)}, \tau \in d u, X(\tau)=j \mid X^{\varepsilon}(0)=i\right)=E\left(e^{-\lambda \xi_{i}^{\varepsilon}(t)}\right) \cdot P\left\{\tau \in d u, X(\tau)=j \mid X^{\varepsilon}(0)=i\right\} .
$$

Denote $P\left\{\tau \in d u, X(\tau)=j \mid X^{\varepsilon}(0)=i\right\}=p_{i j}^{\varepsilon}(d u)$. As result, we obtain multidimensional renewal equation

$E_{i}\left(e^{-\lambda \zeta^{\varepsilon}(t)}\right)=E_{i}\left(e^{-\lambda \zeta_{i}^{\varepsilon}(t)}\right) \cdot P\left\{\tau<t \mid X^{\varepsilon}(0)=i\right\}+\sum_{j=1}^{m} \int_{0}^{t}\left(E_{i}\left(e^{-\lambda \zeta^{\varepsilon}(u)}\right)\right) p_{i j}^{\varepsilon}(d u) \cdot E_{j}\left(e^{-\lambda \zeta^{\varepsilon}(t-u)}\right)$.

The following conditions are true for this renewal equation.

2010 Mathematics Subject Classification: $60 \mathrm{~K} 05$.

Keywords: Markov process; renewal equation; nonlinear approximation; process with independent increments; multidimensional renewal equation.

doi:10.30970/ms.56.1.103-106

(C) O. A. Yarova, Ya. I. Yeleyko , 2021 
1. $E_{i}\left(e^{-\lambda \zeta^{\varepsilon}(u)}\right) p_{i j}^{\varepsilon}(d u) \geq 0, E_{i}\left(e^{-\lambda \zeta^{\varepsilon}(u)}\right) p_{i j}^{\varepsilon}(d u)<\infty$.

2. $E_{i}\left(e^{-\lambda \zeta^{\varepsilon}(u)}\right) p_{i j}^{\varepsilon}(d u)$ is an indecomposable matrix.

3. There is a weak convergence $E_{i}\left(e^{-\lambda \zeta^{\varepsilon}(u)}\right) p_{i j}^{\varepsilon}(d u) \rightarrow E_{i}\left(e^{-\lambda \zeta(u)}\right) p_{i j}(d u)$, when $\varepsilon \rightarrow 0$.

4. $E_{i}\left(e^{-\lambda \zeta(u)}\right) p_{i j}(d u)$ is a block folding matrix.

5. $E_{i}\left(e^{-\lambda \zeta(u)}\right) p_{i j}(d u)$ is uniformly integrable.

Suppose $w_{1} \in E_{1}, w_{2} \in E_{2}, \ldots, w_{r} \in E_{r}, D=\left\{w_{1}, \ldots, w_{r}\right\}$.

Consider the next renewal functions

$$
H_{i j}^{\varepsilon(0)}(t)=E_{i}\left(e^{-\lambda \xi_{i}^{\varepsilon}(t)}\right) \cdot P\left\{\tau<t \mid X^{\varepsilon}(0)=i\right\}+\sum_{s=1}^{r} E_{i}\left(e^{-\lambda \zeta^{\varepsilon}(u)}\right) \cdot p_{i w_{s}}^{\varepsilon}(d u) * E_{w_{s} j}\left(e^{-\lambda \zeta^{\varepsilon}(t-u)}\right)
$$

and

$$
H_{i j}^{\varepsilon(n)}(t)=H_{i j}^{\varepsilon(0)}(t)+\sum_{m \notin D} E_{i}\left(e^{-\lambda \zeta^{\varepsilon}(u)}\right) \cdot p_{i m}^{\varepsilon}(d u) * E_{m j}^{\varepsilon(n-1)}(t), \varepsilon \rightarrow 0 .
$$

Let us introduce the sequence $L_{i j}^{\varepsilon(0)}=E_{i}\left(e^{-\lambda \zeta^{\varepsilon}(u)}\right) \cdot p_{i j}^{\varepsilon}(d u)$. Then

$$
L_{i j}^{\varepsilon(n)}(t)=E_{i}\left(e^{-\lambda \zeta^{\varepsilon}(u)}\right) \cdot p_{i j}^{\varepsilon}(d u)+\sum_{m \notin D} E_{i}\left(e^{-\lambda \zeta^{\varepsilon}(u)}\right) \cdot p_{i m}^{\varepsilon}(d u) \cdot L_{m j}^{\varepsilon(n-1)}(t) .
$$

Hence

$H_{i j}^{\varepsilon(n)}(t)=H_{i j}^{\varepsilon(0)}(t)+\sum_{m \notin D} L_{i m}^{\varepsilon(n-1)}(t) \cdot H_{m j}^{\varepsilon(0)}(t)+\sum_{i=1}^{r} L_{i w_{s}}^{\varepsilon(n)} \cdot E_{i}\left(e^{-\lambda \zeta^{\varepsilon}(u)}\right) p_{i w_{s}}^{\varepsilon}(d u) E_{w_{s} j}\left(e^{-\lambda \zeta^{\varepsilon}(t-u)}\right)$.

Therefore,

$$
H_{w_{s} j}^{\varepsilon}(t)=L_{w_{s} j}^{\varepsilon}(t)+\sum_{n=1}^{r} L_{w_{s} w_{n}}^{\varepsilon} * H_{w_{n} j}^{\varepsilon}(t) .
$$

The solution of this equation can be represented in the next form

$$
E_{i}\left(e^{-\lambda \zeta^{\varepsilon}(t)}\right)=\sum_{n=1}^{k} \int_{0}^{t} E_{i}\left(e^{-\lambda \zeta_{n}^{\varepsilon}(t)}\right) p_{i j}(d u) L_{w_{n} j}^{\varepsilon}(t-u) .
$$

Theorem 1. If $E\left(e^{-\lambda \zeta^{\varepsilon}(u)}\right) p_{i j}(d u)$ is a non-lattice matrix and conditions 1-5 hold, then there exist a matrix $C$ and a normalizing factor $g(\varepsilon) \rightarrow 0(\varepsilon \rightarrow 0)$ such that for $i \in E_{s}, j \in E_{k}$

$$
\lim _{\varepsilon \rightarrow 0} H_{i j}^{\varepsilon}\left[\frac{t}{g(\varepsilon)}, \frac{t}{g(\varepsilon)}+u\right]=u \cdot q_{s k}(t) \cdot \frac{p_{j}^{(k)}}{\pi_{k}}
$$

where $q_{s k}=\left[e^{t C}\right]_{s k}, \pi_{k}=\sum_{i, j \in E_{k}} p_{i}^{(k)} \cdot \int_{0}^{\infty} t F_{i j}(d t), H_{i j}^{\varepsilon}\left[\frac{t}{g(\varepsilon)}, \frac{t}{g(\varepsilon)}+u\right]$ is a renewal function on the interval $\left[\frac{t}{g(\varepsilon)}, \frac{t}{g(\varepsilon)}+u\right]$.

Proof. Suppose $w_{1} \in E_{1}, w_{2} \in E_{2}, \ldots, w_{r} \in E_{r}, D=\left\{w_{1}, \ldots, w_{r}\right\}$ and introduce the next functions

$$
H_{i j}^{\varepsilon(0)}(t)=\delta_{i j}+\sum_{s=1}^{r} E_{i w_{s}}\left(e^{-\lambda \zeta^{\varepsilon}(t)}\right) * H_{w_{s} j}^{\varepsilon}(t),
$$




$$
H_{i j}^{\varepsilon(n)}(t)=H_{i j}^{\varepsilon(0)}(t)+\sum_{m \notin D} E_{i m}\left(e^{-\lambda \zeta^{\varepsilon}(t)}\right) * H_{m j}^{\varepsilon(n-1)(t)} .
$$

We consider

$$
\begin{gathered}
H_{i j}^{\varepsilon}(t)-H_{i j}^{\varepsilon(n)}(t)=\sum_{m_{1} \notin D} E_{i m_{1}}\left(e^{-\lambda \zeta^{\varepsilon}(t)}\right) *\left(H_{m_{1} j}^{\varepsilon}(t)-H_{m_{1} j}^{\varepsilon(n-1)}(t)\right)=\ldots= \\
=\sum_{m_{1} \notin D, \ldots, m_{n} \notin D} E_{i m_{1}}\left(e^{-\lambda \zeta^{\varepsilon}(t)}\right) * . . * E_{m_{n-1} m_{n}}\left(e^{-\lambda \zeta^{\varepsilon}(t)}\right) *\left(H_{m_{n} j}^{\varepsilon}(t)-H_{m_{n} j}^{\varepsilon(0)}(t)\right)= \\
=\sum_{m_{1} \notin D, \ldots, l \notin D} E_{i m_{1}}\left(e^{-\lambda \zeta^{\varepsilon}(t)}\right) * \cdots * E_{m_{n} l}\left(e^{-\lambda \zeta^{\varepsilon}(t)}\right) * H_{l j}^{\varepsilon}(t) \leq E\left(e^{-\lambda \zeta^{\varepsilon(* n)}(t)}\right) * H^{\varepsilon}(t) \rightarrow 0,
\end{gathered}
$$

when $\varepsilon \rightarrow 0$.

So, $H_{i j}^{\varepsilon}(t) \rightarrow H_{i j}^{\varepsilon(n)}(t)$, when $n \rightarrow \infty, \varepsilon \rightarrow 0$.

Arguing as in [1] we deduce

$$
H_{w_{s} w_{k}}^{\varepsilon}\left(\frac{t}{g(\varepsilon)}+y\right)-H_{w_{s} w_{k}}^{\varepsilon}\left(\frac{t}{g(\varepsilon)}\right) \rightarrow y \cdot q_{s k}(t) \cdot \frac{1}{m_{k}},
$$

where $q_{s k}(t)$ is the $(s, k)$-th matrix element.

Then,

$$
\begin{aligned}
H_{w_{s} j}^{\varepsilon}\left(\frac{t}{g(\varepsilon)}+u\right)- & H_{w_{s} j}^{\varepsilon}\left(\frac{t}{g(\varepsilon)}\right)=\sum_{m=1}^{r} \int_{0}^{\frac{t}{g(\varepsilon)}+y} H_{w_{s} w_{m}(d u) L_{w_{m} j}^{\varepsilon}}\left(\frac{t}{g(\varepsilon)}+y-u\right)- \\
& -\sum_{m=1}^{r} \int_{0}^{\frac{t}{g(\varepsilon)}} H_{w_{s} w_{m}}^{\varepsilon}(d u) L_{w_{m} j}^{\varepsilon}\left(\frac{t}{g(\varepsilon)}-u\right) .
\end{aligned}
$$

Integrating by parts we obtain

$$
\begin{gathered}
H_{w_{s} j}^{\varepsilon}\left(\frac{t}{g(\varepsilon)}+u\right)-H_{w_{s} j}^{\varepsilon}\left(\frac{t}{g(\varepsilon)}\right)= \\
=\sum_{m=1}^{r} \int_{0}^{\frac{t}{g(\varepsilon)}}\left[H_{w_{s} w_{m}}^{\varepsilon}\left(\frac{t}{g(\varepsilon)}+y-u\right)-H_{w_{s} w_{m}}\left(\frac{t}{g(\varepsilon)-u}\right)\right] L_{w_{m} j}^{\varepsilon}(d u)+ \\
+\sum_{m=1}^{r} \int_{0}^{\frac{t}{g(\varepsilon)}+y} H_{w_{s} w_{m}}^{\varepsilon}\left(\frac{t}{g(\varepsilon)}+y-u\right) L_{w_{m} j}(d u)-\sum_{m=1}^{r}\left[L_{w_{m} j}^{\varepsilon}\left(\frac{t}{g(\varepsilon)}+y\right)-L_{w_{m} j}\left(\frac{t}{g(\varepsilon)}\right)\right] .
\end{gathered}
$$

We then find the limit

$$
\begin{gathered}
\int_{\frac{t}{g(\varepsilon)}}^{\frac{t}{g(\varepsilon)}+y} H_{w_{s} w_{m}}^{\varepsilon}\left(\frac{t}{g(\varepsilon)}+y-u\right) L_{w_{m} j}^{\varepsilon}(d u)-\left[L_{w_{m} j}^{\varepsilon}\left(\frac{t}{g(\varepsilon)}+y\right)-L_{w_{m} j}^{\varepsilon}\left(\frac{t}{g(\varepsilon)}\right)\right] \leq \\
\leq\left(H_{w_{s} w_{m}}(y)-I\right)\left[L_{w_{m} j}\left(\frac{t}{g(\varepsilon)}+y\right)-L_{w_{m} j}^{\varepsilon}\left(\frac{t}{g(\varepsilon)}\right)\right] \longrightarrow 0, \quad \varepsilon \longrightarrow 0 .
\end{gathered}
$$

Then, for a fixed $T>0$ and we evaluate the next integral

$$
\sup _{\varepsilon} \int_{T}^{\frac{t}{g(\varepsilon)}}\left[H_{w_{s} w_{m}}^{\varepsilon}\left(\frac{t}{g(\varepsilon)}+y-u\right)-H_{w_{s} w_{m}}^{\varepsilon}\left(\frac{t}{g(\varepsilon)}-u\right)\right] L_{w_{m} j}^{\varepsilon}(d u) \leq
$$




$$
\begin{aligned}
\leq \sup _{\varepsilon} & \sum_{r \geq[T / h]} \int_{r h}^{(r+1) h}\left[H_{w_{s} w_{m}}^{\varepsilon}\left(\frac{t}{g(\varepsilon)}+y-u\right)-H_{w_{s} w_{m}}\left(\frac{t}{g(\varepsilon)}-u\right)\right] L_{w_{m} j}^{\varepsilon}(d u) \leq \\
\leq & \sup _{\varepsilon} \sum_{r \geq[T / h]}\left[H_{w_{s} w_{m}}^{\varepsilon}\left(\frac{t}{g(\varepsilon)}+y-r h\right)-H_{w_{s} w_{m}}\left(\frac{t}{g(\varepsilon)}-(r h+h)\right)\right] \times \\
& \times \int_{r h}^{(r+1) h} L_{w_{m} j}^{\varepsilon}(d u) \leq[A(y+h)+2 B] \sup _{\varepsilon} \int_{r h}^{(r+1) h} L_{w_{m} j}^{\varepsilon}(d u) \leq \\
\leq & {[A y+2 B] \cdot \sup _{\varepsilon}\left[L_{w_{m} j}^{\varepsilon}(\infty)-L_{w_{m} j}^{\varepsilon}(T)\right]+A \cdot \sup _{\varepsilon} \int_{T}^{\infty} u L_{w_{m} j}^{\varepsilon}(d u) . }
\end{aligned}
$$

Hence,

$$
\varlimsup_{\varepsilon \rightarrow 0} \sup _{0 \leq u \leq T}\left[H_{w_{s} w_{k}}^{\varepsilon}\left(\frac{t}{g(\varepsilon)}+y-u\right)-H_{w_{s} w_{k}}\left(\frac{t}{g(\varepsilon)}-u\right)\right] \leq A y+2 B<\infty
$$

and from [2] we obtain

$$
\int_{0}^{T}\left[H_{w_{s} w_{k}}^{\varepsilon}\left(\frac{t}{g(\varepsilon)}+y\right)-H_{w_{s} w_{k}}^{\varepsilon}\left(\frac{t}{g(\varepsilon)}\right)\right] L_{w_{k} j}^{\varepsilon}(d u) \longrightarrow y \cdot q_{s k}(t) \cdot \frac{1}{m_{k}} L_{w_{k} j}(t) .
$$

Then

$$
H_{w_{s} j}^{\varepsilon}\left(\frac{t}{g(\varepsilon)}+y\right)-H_{w_{s} j}^{\varepsilon}\left(\frac{t}{g(\varepsilon)}\right) \longrightarrow y \cdot q_{s k} \cdot \frac{1}{m_{k}} L_{w_{k} j}
$$

As result,

$$
H_{i j}^{\varepsilon}\left(\frac{t}{g(\varepsilon)}+y\right)-H_{i j}^{\varepsilon}\left(\frac{t}{g(\varepsilon)}\right) \longrightarrow y \cdot L_{i w_{s}} \cdot q_{s k}(t) \cdot \frac{1}{m_{k}} L_{w_{k} j}
$$

\section{REFERENCES}

1. Ya.I. Yeleyko, I.I. Nishchenko, A limit theorem for a matrix-valued evolution, Visnyk L'viv. Universytety, 53 (1999), 102-107 (in Ukrainian).

2. I.I. Nishchenko, On an asymptotic representation of a normalizing factor for a random matrix-valued evolution, Theor. Probability and Math. Statist., 64 (2002), 153-160.

Ivan Franko National University of Lviv

Lviv, Ukraine

oksana.yarova@lnu.edu.ua

yaroslav.yeleyko@lnu.edu.ua 